\title{
Propensity score to detect baseline imbalance in cluster randomized trials: the role of the c-statistic
}

\author{
Clémence Leyrat ${ }^{1,2,3,4^{*}}$, Agnès Caille ${ }^{1,2,3,5}$, Yohann Foucher ${ }^{6}$ and Bruno Giraudeau $1,2,3,5$
}

\begin{abstract}
Background: Despite randomization, baseline imbalance and confounding bias may occur in cluster randomized trials (CRTs). Covariate imbalance may jeopardize the validity of statistical inferences if they occur on prognostic factors. Thus, the diagnosis of a such imbalance is essential to adjust statistical analysis if required.

Methods: We developed a tool based on the $c$-statistic of the propensity score (PS) model to detect global baseline covariate imbalance in CRTs and assess the risk of confounding bias. We performed a simulation study to assess the performance of the proposed tool and applied this method to analyze the data from 2 published CRTs.

Results: The proposed method had good performance for large sample sizes ( $n=500$ per arm) and when the number of unbalanced covariates was not too small as compared with the total number of baseline covariates ( $\geq 40 \%$ of unbalanced covariates). We also provide a strategy for pre selection of the covariates needed to be included in the PS model to enhance imbalance detection.
\end{abstract}

Conclusion: The proposed tool could be useful in deciding whether covariate adjustment is required before performing statistical analyses of CRTs.

Keywords: Cluster randomized trial, Confounding bias, Propensity score, C-statistic, Baseline imbalance

\section{Background}

In cluster randomized trials (CRTs), the units of randomization are not individuals but rather the social units to which the individuals belong [1]. This may challenge the balance between groups in terms of baseline covariates. Indeed, clusters are sometimes randomized before the identification and recruitment of participants, which may jeopardize allocation concealment [2-5]. In their review, Puffer et al. [6] showed that $39 \%$ of the selected CRTs were at risk of confounding bias on individual characteristics. That was also supported by the work of Brierley et al. [7], who found a risk of bias in $40 \%$ of CRTs that did not use prior identification of participants. In addition, the risk of chance imbalances increases when the number of randomized clusters decreases, which is frequent $[8,9]$.

${ }^{*}$ Correspondence: clemence.leyrat@lshtm.ac.uk

1 INSERM U1153, Paris, France

2 INSERM CIC 1415, Tours, France

Full list of author information is available at the end of the article
Some allocation techniques have been proposed to achieve a better baseline balance in CRTs, but they are not always feasible to implement in practice [10]. If imbalance occurs on one or more prognostic factors, the intervention effect estimate may be biased and could compromise the validity of statistical inferences. Identifying baseline imbalance in CRTs is therefore of importance to implement suitable statistical analyses.

In individually randomized trials, statistical testing is not recommended to assess group comparability because if randomization is properly applied, all observed imbalances will be due to chance [11, 12]. When reporting the results of a randomized trial, the CONSORT statement advises displaying baseline characteristics in a table to gauge group comparability [13]. The same recommendation is given in the CONSORT extension for CRTs, both for individual-level and cluster-level covariates [14]. Fayers and King [15] stated that significance tests "are 
usually only worth doing if potential violation of the randomisation is suspected". In some CRTs, allocation concealment is impossible (i.e., when, for instance, participants are recruited after the randomization of clusters, and because no blinding is possible), and therefore, in this case, tests may be worthwhile. Nevertheless, Wright et al. [16] showed that about $44.7 \%$ of papers reporting the results of a CRT did not provide a statistical test for covariate balance, and $20 \%$ did not even display a table reporting covariates between groups.

The problem of baseline imbalance observed in some CRTs is close to the imbalance that can occur in observational studies [17]. For these latter studies, several methods exist to assess group comparability at baseline. The methods can be divided into two groups: those that assess covariate balance one by one, and those that allow a global assessment of the balance on several baseline covariates [18]. Significance testing (based on $t$ test or $\chi^{2}$ test, for example), standardized difference [19], overlapping coefficient [18] and Kolmogorov-Smirnov [20] or Lévy [21] distances are in the first group of methods. Belister [22] found that the standardized difference (see Table 1) had the highest correlation with the bias of the intervention effect estimate. Standardized differences also perform well with small sample sizes [23], so it may have the best performance in detecting baseline imbalance when covariates are considered one by one. Nevertheless, this method does not provide a global overview of the overlap of covariates between groups. Global assessment of imbalance on several covariates simultaneously is of interest in that it allows for capturing the correlations between covariates.

Table 1 Standardized differences

Baseline groups comparability for each covariate can be assessed with the standardized difference [19]. For a continuous covariates $X$, the standardized difference SD is:

$$
\mathrm{SD}=\frac{100 \times\left|\bar{x}_{1}-\bar{x}_{0}\right|}{\sqrt{\frac{s_{0}^{2}+s_{1}^{2}}{2}}}
$$

where $\bar{x}_{0}$ and $\bar{x}_{1}$ are $X$ means in control and intervention arm, respectively, and $s_{0}^{2}$ and $s_{1}^{2}$ the corresponding variance estimates. For a binary covariate, the SD is expressed as follows:

$$
\mathrm{SD}=\frac{100 \times\left|\hat{P}_{1}-\hat{P}_{0}\right|}{\sqrt{\frac{\hat{P}_{0}\left(1-\hat{P}_{0}\right)+\hat{P}_{1}\left(1-\hat{P}_{1}\right)}{2}}},
$$

where $\hat{P}_{0}$ and $\hat{P}_{1}$ are the observed rates for the covariate in control and intervention arm, respectively. The strength of SD as compared to statistical tests is that this measure does not depend on the sample size nor on the measurement scale [50]. Usually, covariates with a SD exceeding $10 \%$ are considered to be unbalanced [41]. However, for binary covariates, a SD of $10 \%$ can sometimes be negligible [51].
For example, let us consider two quantitative prognostic factors, for which the impact on the outcome is on the same direction: high values for these covariates lead to a higher risk of an event. Because each of these prognostic factors is slightly unbalanced, a univariate test may not detect any imbalance. However, the impact of both imbalances together may cause an important bias in the intervention effect estimate. Consequently, a global approach is more appropriate in the context of CRTs to handle complex relationships underlying a potential confounding bias.

Global metrics include the Mahalanobis distance [24], the post-matching $c$-statistic of the propensity score (PS) model [18] and $L^{1}$ measure [25]. Franklin et al. [18] found that the $c$-statistic of the PS model led to the better prediction of bias for binary, count or continuous outcome, provided the sample size is large enough. This statistic represents the extent to which covariates can predict intervention allocation. The $c$-statistic of the PS model has been used to help in the selection of variables to include in the PS model (even if this method is not recommended [26]) but to our knowledge has not been used as a tool to detect baseline imbalance.

In this context, we developed a decision rule based on the $c$-statistic of the PS model and its expected probability distribution to assess baseline imbalance. This method can be viewed as a global statistical testing at a $5 \%$ significant level. The basic idea is to use the distribution of the c-statistic in accordance with the characteristics of the CRT (size, number of covariates) to choose the cutoff for the detection of imbalance, rather than using a unique threshold value. It is important to note that the PS model fitted in order to detect imbalance is different from the model fitted for the statistical analysis of the trial. In both situation, the outcome of the PS model is the treatment allocation, but, in the former situation, all covariates associated with treatment allocation have to be included in the PS model, whereas in the latter, covariates both linked to treatment allocation and the outcome need to be accounted for. Indeed, when analyzing the trial, selecting confounding only for a propensity score analysis is desirable $[27,28]$ while such a restriction does not hold for our aim which is to detect any baseline imbalance, to obtain a qualitative assessment of the risk of bias in a given CRT. This paper is organized as follows. We first describe two CRTs motivating examples at risk of confounding bias because clusters were randomized before the patients were enrolled. We then give the theoretical background for the PS approach and the $c$-statistic, followed by the objectives of the present paper and the principle of our method. Then, we give the design and the results of a simulation study to assess the performance of our method based on the distribution of the $c$-statistic to detect baseline imbalance in CRTs. The 
implication in terms of risk of confounding bias and need for covariates adjustment are then discussed, along with an application of our method with the two motivating examples.

\section{Motivating examples \\ Example 1: Management of osteoarthritis with a patient-administered assessment tool}

The first motivating example was a published CRT using a $2 \times 2$ factorial design that aimed to assess the impact of an unsupervised home-based exercise programme, the use of standardized evaluation tools, their combination, or usual care on symptoms (pain, global assessment of disease and physical functioning) in patients with knee and hip osteoarthritis (OA) [29]. A total of 867 rheumatologists were randomized and each had to enrol four patients (three with knee OA and one with hip OA). Thus, rheumatologists were not blinded to intervention allocation. For simplicity, we focus on only one intervention: the use of standardized evaluation tools. In all, 1462 patients received the standardised evaluation tools and 1495 patients received usual care. Twelve covariates were collected at baseline (Table 2). Standardized differences are displayed to assess the balance between arms. Univariate statistical testing showed an imbalance in age, pain, disability (measured by the the Western Ontario McMaster University Osteoarthritis Index [WOMAC] physical function subscale) and global assessment of disease at a $5 \%$ significance level. These imbalances correspond to a standardized difference of $7.81 \%$ for age but greater than $10 \%$ for the other variables. Moreover, these variables were known to be strongly associated with the potential outcome of the subjects. Because these variables were associated with whether patients were enrolled into the trial in a given group, they constituted possible confounders. In addition, pain, WOMAC score and global assessment of disease were correlated with each other, with Pearson correlation coefficients in the range [0.38-0.49].

\section{Example 2: Standardized consultation for patients with osteoarthritis of the knee}

The second example was a CRT which evaluated the impact of standardized consultations on patients with OA of the knee versus usual care [30]. It was an open pragmatic CRT in which 198 rheumatologists were randomized, each of whom had to include two consecutive patients who met the inclusion criteria. In total, 154

Table 2 Patient baseline characteristics per group in the study on management of osteoarthritis with a standardized evaluation tool (first motivating example)

\begin{tabular}{|c|c|c|c|c|}
\hline Characteristics & $\begin{array}{c}\text { Control } \\
n_{0}=1495\end{array}$ & $\begin{array}{c}\text { Standardized tool } \\
\qquad n_{1}=1462\end{array}$ & $p$ & SDiff ( \%) \\
\hline & mean (standard deviation) & mean (standard deviation) & & \\
\hline Duration of symptoms (months) & $68.0(69.8)$ & $70.9(75.0)$ & 0.2737 & 4.03 \\
\hline Age (years) & $67.2(9.7)$ & $66.4(10.0)$ & 0.0344 & 7.81 \\
\hline $\mathrm{BMI}\left(\mathrm{kg} \cdot \mathrm{m}^{-2}\right)$ & $27.8(4.9)$ & $27.7(4.7)$ & 0.3824 & 3.14 \\
\hline Patient global assessment (0-100) & $61.1(18.2)$ & $56.7(17.4)$ & $<0.0001$ & 24.44 \\
\hline Pain evaluation VAS (0-100) & $59.4(16.0)$ & $55.3(15.1)$ & $<0.0001$ & 26.32 \\
\hline \multirow[t]{2}{*}{ WOMAC function score (0-100) } & $45.5(16.3)$ & $43.8(16.0)$ & 0.0050 & 10.32 \\
\hline & $n(\%)$ & $n(\%)$ & & \\
\hline Osteoarthritis in other joints & $1366(91.4)$ & $1311(89.7)$ & 0.1297 & 5.81 \\
\hline \multicolumn{5}{|l|}{ Prior treatment } \\
\hline IA treatment & $434(29.0)$ & $432(29.6)$ & 0.7877 & 1.14 \\
\hline NSAIDs & $955(63.9)$ & $958(65.5)$ & 0.3689 & 3.45 \\
\hline SYSADOA & $617(41.3)$ & $605(41.4)$ & 0.9810 & 0.22 \\
\hline Male & $424(28.4)$ & $459(31.4)$ & 0.0782 & 6.65 \\
\hline Kellgren and Lawrence grade & & & 0.2594 & \\
\hline III & $724(48.4)$ & $677(46.3)$ & & 4.25 \\
\hline IV & $516(34.5)$ & $547(37.4)$ & & 6.02 \\
\hline
\end{tabular}

BMI: Body Mass Index; VAS: Visual Analogue Scale; WOMAC: Western Ontario and McMaster Universities Arthritis Index; IA: intra-articular; NSAID: non-steroidal anti-inflammatory drug; SYSADOA: systematic slow acting drug for osteoarthritis. SDiff: standardized difference; $p$ : $p$-value for univariate tests (adjusted $t$ test for quantitative variables, adjusted chi-square test for qualitative variables to take the clustering into account). Bold values are significant tests at a $5 \%$ significance level 
patients were allocated to standardized consultation and 182 to usual care. Overall, 26 covariates were measured at baseline (Table 3). Statistical testing revealed a significant imbalance in body mass index (BMI), delay in years since the beginning of pain, age at the beginning of pain and the use of non-drug treatments. Moreover, some other variables (weight, pain, Medical Outcomes Study Short Form 12 [SF-12] mental, and eight concommitant treatments) had a standardized difference greater than the usual threshold of $10 \%$.

\section{Theoretical background \\ Propensity score theory}

The PS theory was initially developed by Rosenbaum and Rubin [31] to overcome the problem of confounding bias in observational studies. The individual PS refers to the individual probability, for a subject $l$ involved in a study, of receiving the intervention of interest $\left(T_{l}=1\right)$ rather than the control intervention $\left(T_{l}=0\right)$, conditionally on the subject's characteristics at baseline $x_{l}=\left(x_{(1) l}, \ldots, x_{(r) l}\right)$. The PS is frequently denoted by $e\left(x_{l}\right)$ and is defined as

Table 3 Patient baseline characteristics per group in the study on standardized consultation for patients with osteoarthritis of the knee (second motivating example)

\begin{tabular}{|c|c|c|c|c|}
\hline \multirow[t]{2}{*}{ Characteristics } & Control & Standardized consultation & $p$ & SDiff ( \%) \\
\hline & $n_{0}=146$ & $n_{1}=181$ & & \\
\hline & mean (standard deviation) & mean (standard deviation) & & \\
\hline Age (years) & $64.5(8.4)$ & $63.9(8.1)$ & 0.4720 & 8.02 \\
\hline Weight (kg) & $81.4(13.6)$ & $84.1(12.9)$ & 0.0665 & 20.60 \\
\hline BMI $\left(\mathrm{kg} \cdot \mathrm{m}^{-2}\right)$ & $30.2(3.8)$ & $31.2(3.5)$ & 0.0143 & 27.63 \\
\hline PEL (0-5) & $2.2(0.9)$ & $2.2(0.8)$ & 0.9594 & 0.00 \\
\hline Delay since beginning of pain (years) & $5.5(5.9)$ & $7.4(7.5)$ & 0.0152 & 27.57 \\
\hline Age at beginning of pain (years) & $59.1(10.4)$ & $56.5(10.5)$ & 0.0300 & 24.28 \\
\hline Pain (0-10) & $5.6(1.3)$ & $5.5(1.2)$ & 0.3646 & 10.38 \\
\hline WOMAC function score (0-100) & $29.9(12.2)$ & $30.3(11.7)$ & 0.7377 & 3.76 \\
\hline SF-12 physical subscale & $34.8(6.7)$ & $35.4(6.7)$ & 0.4385 & 8.70 \\
\hline SF-12 mental subscale & $41.4(9.4)$ & $43.3(10.1)$ & 0.0827 & 19.31 \\
\hline \multirow[t]{2}{*}{ Global assessment of disease status (0-10) } & $5.6(1.5)$ & $5.6(1.5)$ & 0.9133 & 1.31 \\
\hline & $n(\%)$ & $n(\%)$ & & \\
\hline Male & $49(27.1)$ & $34(23.3)$ & 0.5132 & 8.73 \\
\hline \multicolumn{5}{|l|}{ Prior treatments } \\
\hline Analgesics & $130(71.8)$ & $96(65.8)$ & 0.2889 & 13.13 \\
\hline NSAIDs & $95(52.5)$ & $90(61.6)$ & 0.1215 & 18.58 \\
\hline Current use of NSAIDS & $160(88.4)$ & $126(86.3)$ & 0.6883 & 6.31 \\
\hline SYSADOA & $74(40.9)$ & $68(46.6)$ & 0.3576 & 11.49 \\
\hline Current use of SYSADOA & $179(98.9)$ & $145(99.3)$ & 1.0000 & 4.46 \\
\hline IA treatment & $31(17.1)$ & $29(19.9)$ & 0.6229 & 7.05 \\
\hline Non-drug treatment & $110(60.8)$ & $71(48.6)$ & 0.0372 & 24.58 \\
\hline Diet & $49(27.1)$ & $31(21.2)$ & 0.2750 & 13.67 \\
\hline Dietetician & $12(6.6)$ & $7(4.8)$ & 0.6401 & 7.91 \\
\hline Physical exercice & $44(24.3)$ & $27(18.5)$ & 0.2571 & 14.22 \\
\hline Physiotherapy & $30(16.6)$ & 17 (11.6) & 0.2692 & 14.20 \\
\hline Knee orthosis & $21(11.6)$ & $11(7.5)$ & 0.2967 & 13.86 \\
\hline Insoles & $24(13.3)$ & $11(7.5)$ & 0.1376 & 18.84 \\
\hline Walking sticks & $13(7.2)$ & $10(6.8)$ & 1.0000 & 1.30 \\
\hline
\end{tabular}

BMI: Body Mass Index; PEL: Baecke's physical exercice level scale; WOMAC: Western Ontario and McMaster Universities Arthritis Index; SF-12: 12-items Short Form; IA: intra-articular; NSAID: non-steroidal anti-inflammatory drug; SYSADOA: systematic slow acting drug for osteoarthritis. SDiff: standardized difference; $p$ : $p$-value for univariate tests (adjusted $t$ test for quantitative variables, adjusted chi-square test for qualitative variables to take the clustering into account). Bold values are significant tests at a $5 \%$ significance level 
$e\left(\boldsymbol{x}_{\boldsymbol{l}}\right)=P\left(T_{l}=1 \mid \boldsymbol{x}_{\boldsymbol{l}}\right)$. The true PS is unknown in practice, but it can be estimated by logistic regression, modeling the probability of receiving the intervention of interest given $r$ observed covariates as follows:

$$
e\left(\boldsymbol{x}_{\boldsymbol{l}}\right)=\left\{1+\exp \left(-\alpha_{0}-\sum_{p=1}^{r} \alpha_{p} x_{(p) l}\right)\right\}^{-1}
$$

where $\alpha_{0}$ is the intercept and $\alpha_{p}(p=1, \ldots, r)$ are the regression coefficients.

In CRTs, the PS has been studied for the estimation of the intervention effect $[27,28]$, or to improve randomization [32] but not for detection of imbalance between groups.

\section{The c-statistic}

The $c$-statistic (concordance statistic) measures the discriminatory capacity of a predictor [33]. It also corresponds to the area under the receiver operating characteristic (ROC) curve, which displays sensitivity as a function of 1specificity for all the possible thresholds of the predictor [34]. If we consider an intervention allocation (intervention $v s$. control), the $c$-statistic is the probability that a subject receiving the intervention has a higher value for the predictor than a subject in the control group [35]. It can be estimated as follows:

$$
c=\frac{1}{M} \sum_{i=1}^{n_{0}} \sum_{j=1}^{n_{1}} \mathbb{1}\left\{p_{i}<p_{j}\right\}
$$

where $i=1, \ldots, n_{0}$ is the participant index in the untreated group, $j=1, \ldots, n_{1}$ is the participant index in the treated group and $\mathbb{1}$ is a dummy variable equals to 1 if $p_{i}<p_{j}, 0$ otherwise. The $c$-statistic takes its values in the range $[0.5 ; 1.0]$, where 0.5 corresponds to a classification that does not outperform chance and 1.0 corresponds to perfect classification. In our situation, the groups are the treatment arms and the predictor is the prediction obtained from the PS model. The $c$-statistic is often computed with the predictions obtained from a logistic model.

\section{Propensity scores and the c-statistic}

In the absence of baseline imbalance, the PS has a normal distribution of mean 0.5 in each group of the study, and thus the baseline variables are independant from the intervention allocation. In other words, the c-statistic of the PS model (3) is close to 0.5. By contrast, if at least one covariate is associated with intervention allocation, the $c$-statistic will be larger than 0.5 . To our knowledge, the $c$-statistic of the PS model has not been used as a tool to detect baseline imbalance.

\section{Objectives and principles}

We developed a method, based on the c-statistic of the PS model, to detect baseline imbalance between groups in CRTs. In practice, this method is a tool to appreciate the risk of confounding bias and to identify the situations in which suitable statistical methods to take imbalance into account must be implemented. Our method relies on three steps:

(i) The $c$-statistic is estimated from the data of the CRT for which one wants to assess the baseline balance

(ii) The $95^{\text {th }}$ percentile of the $c$-statistic distribution under the hypothesis of no systematic baseline imbalance is determined from simulation with the same number of covariates and sample size in the CRT

(iii) The statistical decision rule is expressed as follows: if the $c$-statistic estimated in step (i) is above the threshold value obtained in step (ii), then a baseline imbalance is suspected.

Because of the use of the $95^{\text {th }}$ percentile of the $c$-statistic distribution as a threshold, our method is similar to a global statistical test for baseline imbalance at a $5 \%$ significance level. It is important to note that our method focuses only on individual-level characteristics; indeed, in CRTs, clusters are the unit of randomization and thus any observed imbalance in cluster-level covariates will be due to sampling fluctuations. Applying this method to cluster-level covariates would be similar to baseline tests for individually randomized trials, which is not recommended in practice. Conversely, because participants are not the randomization units in CRTs, confounding bias may affect some trials, leading to systematic imbalances in individual-level variables [17]. Because threshold values are different for each combination of sample size and number of covariates involved (illustrative results are given in Additional file 1), our approach is more flexible than using a unique threshold for the c-statistic. Indeed, our methods uses the empirical distribution of the c-statistic of the PS model considering the characteristics of the CRT of interest.

The objectives of the present paper are to assess the performance of this method with a simulation study and to interpret the diagnosis of baseline imbalance in terms of risk of confounding bias and need for covariate adjustment.

\section{Methods \\ Design of the simulation study}

We performed a simulation study to assess the performance of the proposed method to detect baseline imbalance. The determination of thresholds values for our 
methods (step (ii) in the principle of our method) is described in Additional file 1: Appendix A.

\section{Data generation}

We generated datasets corresponding to CRTs without systematic imbalance and estimated the $c$-statistic of the PS model for each dataset. The data were generated as follows:

- Cluster size: Let us consider a two-parallel-arm CRT, in which $2 k$ clusters of mean size $m$ are randomized. We generated cluster sizes, as proposed by Turner et al. [36], from a Poisson distribution with parameter $m: m_{i j} \sim \mathcal{P}(m),(i=0,1$ the intervention index and $j=1, \ldots, k$ the cluster index).

- Covariates: Let $\boldsymbol{X}=\left(X_{1}, \ldots, X_{r}\right)$ be a vector of $r$ randomly generated covariates, among which $r_{c}$ are continuous covariates and $r_{b}$ are binary $\left(r_{c}+r_{b}=r\right)$. To generate $\boldsymbol{X}$, a vector $\boldsymbol{X}_{\mathbf{0}}$ was first drawn in a multivariate normal distribution $\mathcal{N}_{r} \sim\left(0, \boldsymbol{\Sigma}_{r \times r}\right)$, without loss of generalizability.

At this stage, we have a matrix $\boldsymbol{X}_{\mathbf{0}}$ of $r$ continuous balanced covariates measured at baseline. However, this situation does not differ from an individually randomized trial. To fit to the situation of a real CRT, we induced an intraclass correlation for the covariates meaning that subjects belonging to the same cluster had more similar individual characteristics. We randomly drew a cluster effect $\gamma_{p j},(j=1, \ldots, k)$ for each cluster $j$ and each covariate $p(p=1, \ldots, r)$ in a distribution $\mathcal{N}(0,0.15)$, with the constraint $\sum_{j=1}^{k} \gamma_{p j}=0$ in each arm. The variance parameter for the cluster effect of 0.15 was chosen to obtain intraclass correlation coefficient (ICC) values for the covariates in the range [0.01;0.05]. These values are based on those observed for baseline characteristics in the study of Kul et al. [37]. Then, for each subject in cluster $j$ and each covariate, a random error was drawn from a distribution $\mathcal{N}\left(\gamma_{p j}, 1\right)$ and this error term was added to $X_{\mathbf{0}}$, the initial value of the covariate.

Among the $r$ generated covariates, we induced an imbalance on $s$ of them. These covariates were correlated with each other, because such correlations are often observed in clinical trials [38]. Moreover, for each of the $s$ unbalanced covariates, the standardized difference (reflecting the imbalance 'size') depended on the degree of correlation between covariates: two highly correlated covariates had similar standardized differences. To induce the correlations between the standardized differences, a vector $\boldsymbol{X}_{\mathbf{0}}=\left(X_{1}, \ldots, X_{r}\right)$ of $r$ covariates was first randomly drawn from a multivariate normal distribution $\mathcal{N}_{r} \sim\left(0, \Sigma_{r \times r}\right)$ with the following covariance matrix:
$\Sigma_{r \times r}=\left(\begin{array}{ccccccc}1 & \sigma_{1,2} & \cdots & \sigma_{1, s} & \sigma_{1, s+1} & \cdots & \sigma_{1, r} \\ \sigma_{2,1} & 1 & \cdots & \sigma_{2, s} & \sigma_{2, s+1} & \cdots & \sigma_{2, r} \\ \vdots & \vdots & \ddots & \vdots & \vdots & \cdots & \vdots \\ \sigma_{s, 1} & \sigma_{s, 2} & \cdots & 1 & \sigma_{s, s+1} & \cdots & \sigma_{s, r} \\ \sigma_{s+1,1} & \sigma_{s+1,2} & \cdots & \sigma_{s+1, s} & 1 & \cdots & \sigma_{s+1, r} \\ \vdots & \vdots & \cdots & \vdots & \vdots & \ddots & \vdots \\ \sigma_{r, 1} & \sigma_{r, 2} & \cdots & \sigma_{r, s} & \sigma_{r, s+1} & \cdots & 1\end{array}\right)$.

$\sigma_{f, g}, f, g=1, . ., q, f \neq g$ represents the covariance and the correlation between covariates $X_{f}$ and $X_{g}$ in that the covariates followed standard normal distributions. The covariance matrix $\boldsymbol{\Sigma}_{\boldsymbol{r} \times \boldsymbol{r}}$ was a positive definite matrix randomly generated with the $\mathrm{R}$ function genPositiveDefMat from the clusterGeneration package. For convenience, we considered the absolute values of the covariance matrix.

Second, the sub-matrix $\boldsymbol{\Sigma}_{\boldsymbol{s} \times \boldsymbol{s}}$ of $\boldsymbol{\Sigma}_{\boldsymbol{r} \times \boldsymbol{r}}$ was used to draw the standardized differences for unbalanced covariates from a multivariate normal distribution. Let $\Delta$ and $s_{\Delta}$ be respectively the mean standardized differences of unbalanced covariates and its standard deviations. Thus, the $s$ unbalanced covariates followed a distribution $\mathcal{N}\left(\Delta, s_{\Delta}^{2}\right)$. As $\sigma_{f, g}=r_{f, g} \times$ $\sigma_{j} \sigma_{k}$, the covariance matrix $\boldsymbol{\Sigma}_{\boldsymbol{s} \times \boldsymbol{s}}^{\boldsymbol{\Delta}}$ used to generate the standardized differences was: $\boldsymbol{\Sigma}_{\boldsymbol{s} \times \boldsymbol{s}}^{\boldsymbol{\Delta}}=s_{\Delta}^{2} \boldsymbol{\Sigma}_{\boldsymbol{s} \times \boldsymbol{s}}$. Thus, standardized differences $\boldsymbol{\Delta}=\left(\Delta_{1}, \ldots, \Delta_{s}\right)$ were drawn from a multivariate normal distribution with mean $\boldsymbol{\Delta} \mathbb{1}_{s}$ with $\boldsymbol{\Sigma}_{\boldsymbol{s} \mathbf{x} \boldsymbol{s}}^{\boldsymbol{\Delta}}$ for the covariance matrix. Then, for an unbalanced covariate $f(f=1, \ldots, s)$ and a subject $l$, the covariate's value was $\boldsymbol{X}_{\mathrm{fl}}+\boldsymbol{\Delta} \times T_{l}$, where $\boldsymbol{X}_{\mathrm{fl}}$ corresponded to the $f^{\text {th }}$ covariate's value for subject $l$ when generating $X_{0}$ and $T_{l}$ being the intervention indicator for subject $l$, as previously defined.

Finally, $r_{b}$ covariates from $\boldsymbol{X}_{\mathbf{0}}$ were dichotomized by covariate-specific threshold values $t_{p}\left(p=1, \ldots, r_{b}\right)$. Thresholds $t_{p}$ were a priori fixed to obtain the desired prevalences $P_{p}$ of these characteristics, drawn in a uniform distribution in the range [0.2; 0.8]. From $P_{p}$, the threshold was: $t_{p}=\Phi^{-1}\left(1-P_{p}\right)$, where $\Phi$ is the cumulative density function (CDF) of a standard normal distribution. Doing so, the standardized difference for binary covariates could be calculated from the formula in Table 1 with $\hat{P}_{1_{b}}=\Phi\left(\Phi^{-1}(1-\right.$ $\left.\left.\hat{P}_{0_{b}}\right)-\Delta / 100\right)$, where $\hat{P}_{0 b}$ and $\hat{P}_{1 b}$ are the observed proportions in the control and the intervention arms, respectively.

\section{Propensity score estimation}

The PS was estimated with a logistic model adjusted on the set of generated covariates. A cluster-specific random 
effect cannot be taken into account in this model because clusters are nested in the intervention arm (subjects from the same cluster received the same intervention). Even if this limitation can have an impact when PS is used to estimate the intervention effect [28], the impact on the performance for imbalance detection is negligible because clusters are the unit of randomization. Thus, considering clusters as a fixed effect, cluster effect would be balanced between groups.

\section{Covariate pre-selection}

Within the simulation, we also proposed two criteria to select only some covariates among the $r$ generated covariates, in order to assess the efficiency of a more parsimonious model to detect imbalance, because numerous studies showed the importance of covariate selection for PS model to avoid over-fitting problems [39, 40]. Moreover, the presence of a large number of balanced covariates in the PS model can attenuate the importance of a potential global imbalance. A covariate was included in the PS model if it satisfied at least one of these two criteria:

- its standardized difference was $\geq 5 \%$

- its standardized difference was $<5 \%$ but its correlation with at least one covariate with a standard difference $\geq 5 \%$ was greater or equal to 0.2 in absolute value.

These criteria allowed for selecting covariates with more flexibility than with univariate testing. In practice, a covariate is supposed to be unbalanced when its standardized difference $\geq 10 \%$ [41], whereas our method was less stringent for the number of covariates kept. Moreover, a balanced covariate highly correlated with an unbalanced one may have an impact on the c-statistic. This strategy allowed to assess if baseline imbalance must be diagnosed from all available baseline covariates.

\section{Threshold value}

For each studied scenario, the corresponding threshold value to conclude to baseline imbalance was obtained from simulations with the same simulation parameters but under the hypothesis of no systematic imbalance (i.e. the $r$ generated covariates are balanced). The impact of sample size, number of clusters, number of covariates and trial design (CRT or individually randomized trial) on the $c$-statistic of the PS model without systematic imbalance was studied beforehand and results are presented in Additional file 1: Appendix A.

\section{Results assessment}

The results were assessed in terms of the following:

- proportion $\pi$ of simulated datasets in which the estimated $c$-statistic was greater or equal to the threshold value defined as the 95th percentile of the $c$-statistic distribution in absence of systematic baseline imbalance, i.e., the proportion of situations in which baseline imbalance was detected, according to our proposed rule,

- for each unbalanced covariate, the proportion of significant univariate tests at a $5 \%$ significance level. These tests were adjusted $t$ test and adjusted chi-square test, described in [1] to take the clustering into account.

\section{Studied scenarios}

First, we studied 144 scenarios corresponding to the different combination of the following parameters:

- the sample size per arm: $n=(100,500)$. In CRTs, the median number of subjects per arm is 329 (interquartile range [143-866]) [42]. Thus, the chosen values correspond to the situation of a small and average size CRT.

- the number of clusters per arm: $k=(5,10,50)$,

- the number of covariates: $r=(4,10,20)$ for $n=100$ and $r=(10,20,50)$ for $n=500$, corresponding to ratios $\frac{n}{r}=(25,10,5)$ for $n=100$ and $\frac{n}{r}=(50,25,10)$ for $n=500$. We considered $r_{c}=r_{b}=\frac{r}{2}$.

- the number of unbalanced covariates: $s$ was defined such that the percentage of unbalanced covariates among all covariates was $20 \%$ or $40 \%$ (except for the case $k=5, m=20$ in which $25 \%$ and $50 \%$ of covariates were unbalanced). Thus, $s=(2,4)$ for $r=10, s=(4,8)$ for $r=20$ and $s=(10,20)$ for $r=50$. Among unbalanced covariates, $\frac{s}{2}$ were binary and $\frac{s}{2}$ were continuous.

- the standardized difference for unbalanced covariates: $\Delta\left(s_{\Delta}\right)=10 \%(5 \%)$ or $20 \%(10 \%)$.

Second, we studied the performance of our method after covariate selection according to the rule expressed in Covariate pre-selection section of the main paper. We focused on scenarios in which the total number of covariates was $\geq 20$ and the standardized difference for unbalanced covariates was moderate (10\%), corresponding to 36 different scenarios.

In both situations, we performed 5000 simulations.

\section{Results}

\section{Results without covariate pre-selection}

The results are displayed in (Fig. 1). As expected, the imbalance detection rate $\pi$ (i.e. the proportion of situations in which our method allowed to detect imbalance) was higher when the standardized differences for the unbalanced covariates was high (20\%) than for a moderate imbalance (10\%). Second, imbalance was detected more 


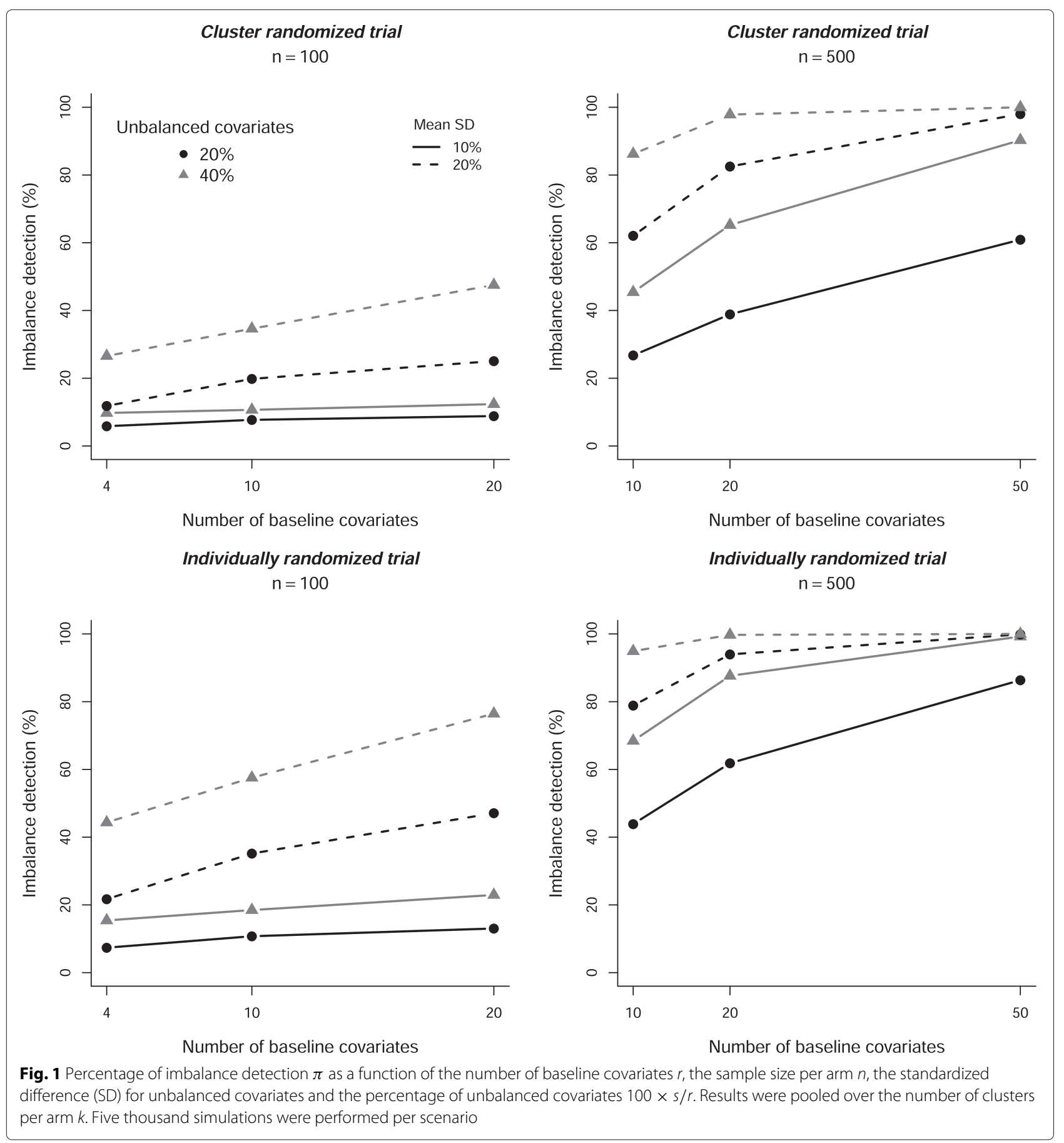

often when the proportion of unbalanced total baseline covariates was higher 40 or $50 \%$ (for $k=5$ and $m=20$ ) than when this proportion was 20 or $25 \%$ (for $k=5$ and $m=20$ ). This result suggested that when there were too many balanced covariates, the information on unbalanced covariates was attenuated.

Moreover, the percentage imbalance detection was higher with sample size $n=500$ than with $n=100$. However, this latter situation corresponded to a small sample size (lower than the first quartile of the sample size per arm in a review of CRTs). This percentage increased also with the number of covariates. When the percentage of unbalanced covariates remained constant, the performance was better with increased number of total covariates (and thus the number of unbalanced ones), which suggests that the method allowed for capturing a global imbalance rather than imbalance on isolated covariates. 


\section{Results with covariate pre-selection}

For a set of 20 baseline covariates, the average number of covariates retained after the pre-selection was 13.5 with $20 \%$ unbalanced covariates and 14.1 with $40 \%$ unbalanced covariates. For a set of 50 baseline covariates, the average number of covariates retained was 27.1 and 29.7 with 20 and $40 \%$ of imbalance, respectively. So this preselection mechanism allowed for retaining a large set of covariates, which was the basic idea for our method.

Moreover, this pre-selecting strategy for the covariates allowed for a systematic improvement in the percentage of bias detection for each study scenario, as displayed in Fig. 2. The relative improvement (defined as the ratio of the difference in percentage of imbalance detection with and without selection) varied from $0.7 \%$ for a scenario in which the initial percentage of imbalance detection equaled $99.4 \%$ before pre-selection, to $116.4 \%$ for the scenario showing the worst performance without covariate pre-selection. However, this strategy was mainly helpful for scenarios in which the initial performance was moderate (about $50 \%$ ). Even after an average improvement $>100 \%$ for a small sample size $(n=100)$, the performance remained $<50 \%$. Indeed, in these situations the risk of chance imbalance due to sampling fluctuations is high (balance is achieved according the law of large numbers). Thus, threshold values for these trials are large even with no systematic imbalance and consequently, the detection rate is small. However, covariate selection increases detection rate in every scenario, so these results confirmed the need for a parsimonious PS model (i.e. including only a subset of covariates) that could be obtained with our simple and automatic proposed strategy.

\section{From global imbalance to confounding bias}

Once the imbalance is detected, further assessment could be conducted to assess any risk of confounding bias, that is, if at least one of the covariates included in the PS model is also associated with the outcome. Such a variable, known as a confounding factor, is both associated with the intervention allocation and the outcome and may lead to a mis-estimation of the intervention effect [43]. Statistical measures of association can be used to identify them, as well as the literature to identify known confounders for a given outcome. When confounding bias is suspected, adjustment is required, whereas if the imbalance results from chance, adjustment would only improve the precision of the estimate, at least in linear models [44]. Among adjustment methods available for CRTs, multivariable regression [45] or PS-based methods $[46,47]$ are commonly used. However, the best predictive PS model is not the best model to correct imbalance

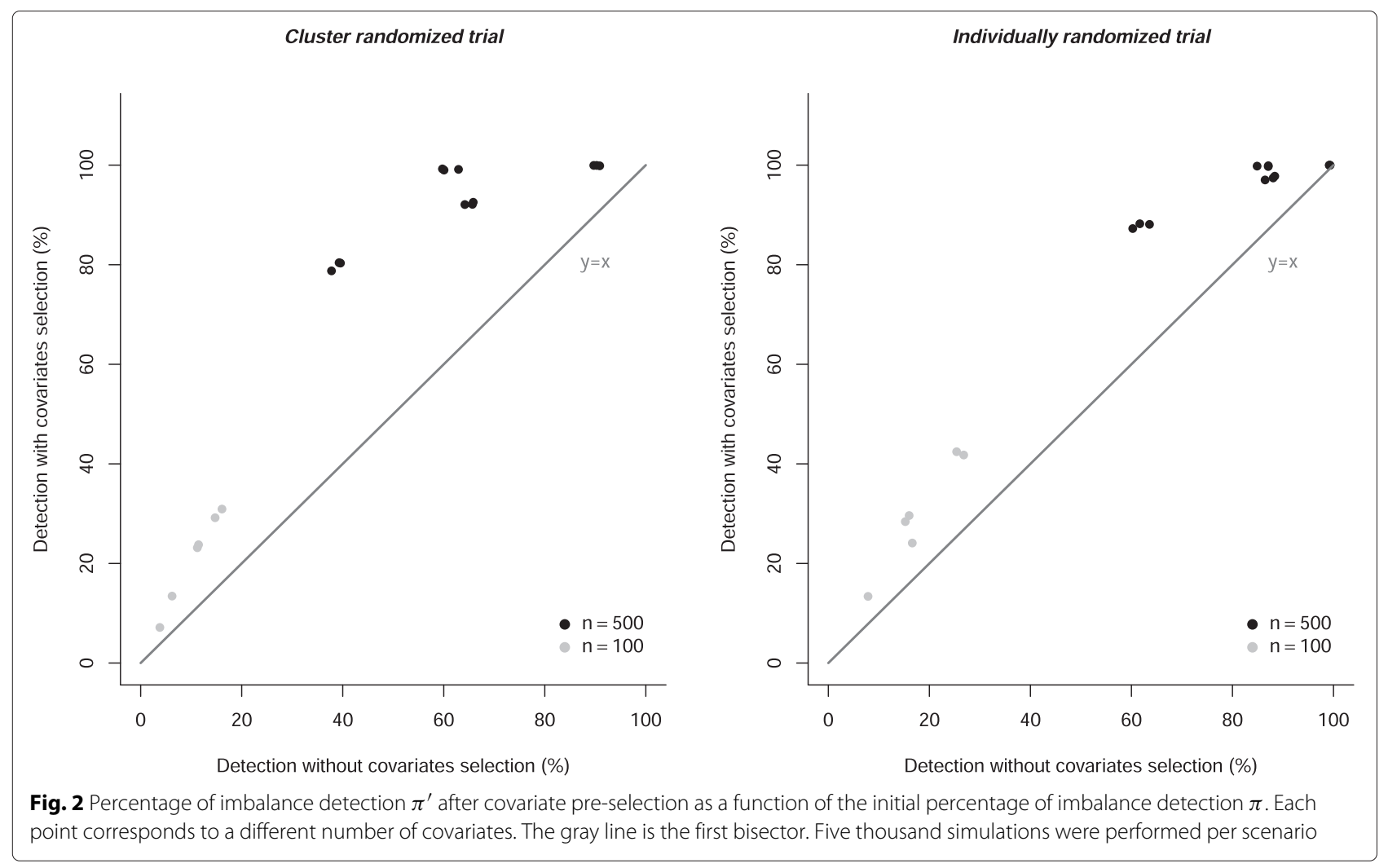


[40]. As compared with a model for imbalance detection which can involve a large amount of covariates, a good PS model would include only confounding factors [39]; covariates which are related only to the intervention would increase standard errors without reducing bias [48]. A simulation study showed that discrimination criteria, such as the $c$-statistic or adequation tests such as Hosmer and Lemeshow cannot detect the omission of a confounding factor [26]. Consequently, the model built to detect imbalance is not the most proper for the statistical analysis.

Figure 3 displays the different steps that help identifying the need of covariate adjustment. If patients are identified before cluster randomization and if the sample size is large enough, there is no risk of global imbalance or confounding bias and adjustment is not required. If cluster randomization occurs after patients recruitment but the sample size is small, there is a risk of chance imbalance. If cluster randomization occurs beforehand, there is a risk of systematic bias. In the last two situations, our tool can detect a global imbalance. If a such imbalance is detected, the assessment of the association between covariates and the outcome is needed to identify confounding bias, i.e. the presence of covariates both linked to the intervention and the outcome. When confounders are detected, covariate adjustment is needed to obtain an unbiased estimate of intervention effect. Otherwise, covariate adjustment will have no impact on the estimate but can increase precision for linear models.

\section{Results from the two motivating examples}

For the two following examples, threshold values to detect baseline imbalance were obtained under a hypothesis of no systematic imbalance, with the same number of covariates (and the same proportion of continuous and binary covariates) and the same sample size as in the original CRT. For covariate generation, we used the observed mean (or rate) and standard deviations of covariates in the control arm and the correlation matrix from each CRT.

\section{Example 1: Management of OA with a patient-administered assessment tool}

The PS was estimated with a logistic model adjusted on the 12 covariates displayed in Table 2 . The PS distributions

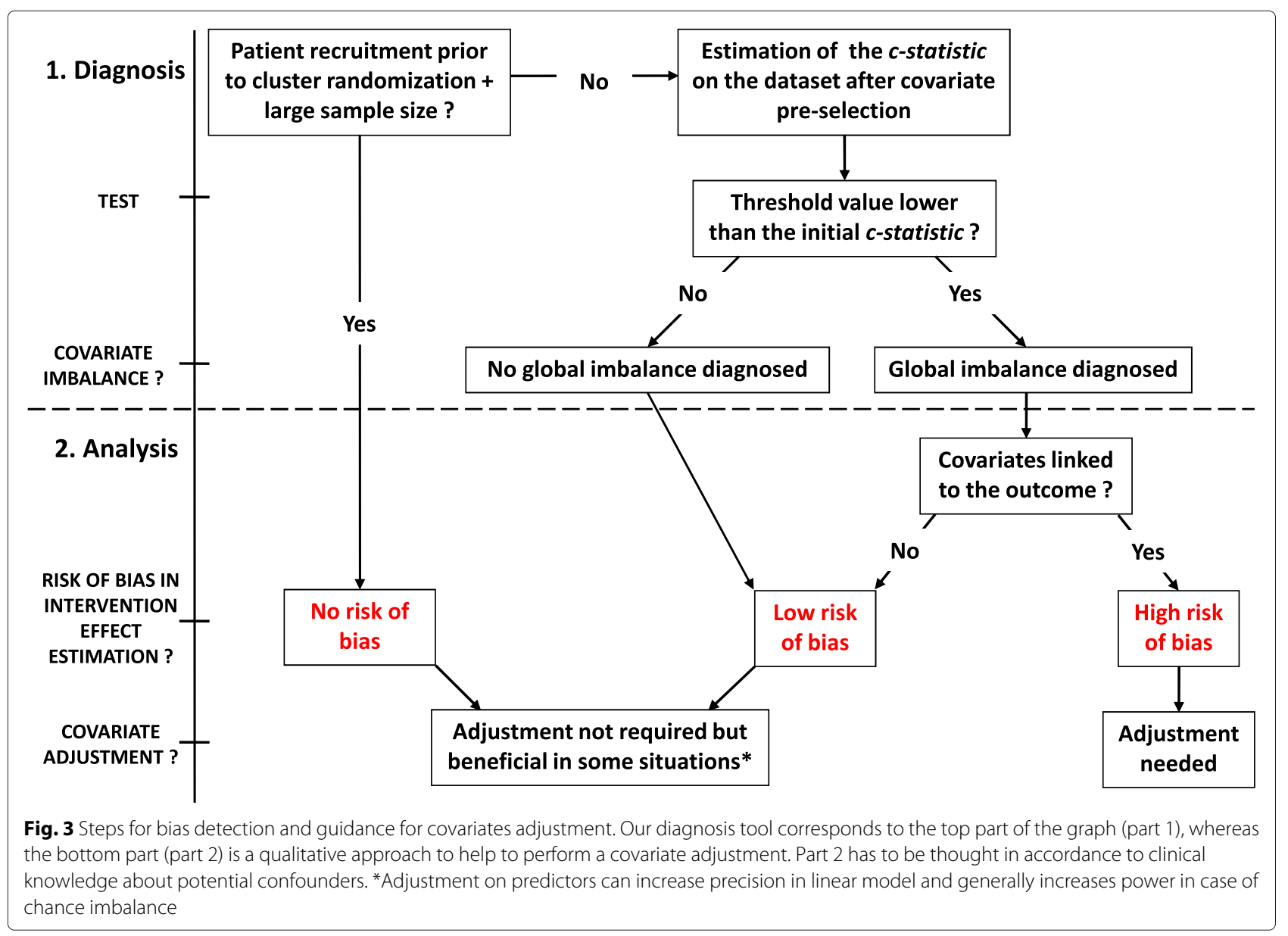


by arm are displayed in Additional file 1: Appendix C Figure $2 \mathrm{a}$. The estimated $c$-statistic from this model was 0.598 . The threshold value under the hypothesis of no systematic baseline imbalance was 0.549 , below the estimated c-statistic for the dataset. We also applied our method using the pre-selection methods for the covariates: seven covariates among the 12 measured at baseline were retained. The estimated c-statistic was 0.595 , and the corresponding threshold value was 0.541 . Thus, our method allowed for diagnosing a baseline imbalance, with or without selection for covariates, and highlights the need for adjusted statistical methods. We showed in a previous work a huge difference in the intervention effect estimate obtained from a crude analysis (without adjustment) and that obtained with multivariable regression or PS adjustment [27], and therefore confirmed that baseline imbalance occured on counfounding factors.

Moreover, the results showed that covariates significantly associated with the intervention allocation in the PS model were not the same covariates that appeared significantly unbalanced with univariate tests. Indeed, polyarthritis and radiological grade were significant in the PS model at a $5 \%$ significance level, whereas the WOMAC score was no longer significant. These results were explained by the correlation patterns between covariates, which suggests that global approaches for the diagnosis of baseline imbalance may add some global information on relationships among covariates, missing with the univariate approach.

\section{Example 2: Standardized consultation for patients with $O A$ of the knee}

The PS model was built from the 26 covariates described in Table 3. The PS distribution in the two arms was not layered (see Additional file 1: Appendix C Figure $2 \mathrm{~b})$. However, the estimated $c$-statistic was 0.684 and the threshold value was 0.696 , so the method did not detect imbalance between groups. This situation is close to the case in which the number of unbalanced covariates was small as compared to the total number of covariates, and thus, a pre-selection of covariates is needed. Therefore, we applied the selection strategy previously proposed. From Table 3, Five covariates had a standardized difference $<5 \%$ (physical exercise level [PEL] scale, WOMAC score, global assesment of the disease, current use of SYSADOA and use of walking sticks). Then, we estimated the correlation matrix (Pearson's correlation coefficients were used, both for qualitative and quantitative covariates). Among the five balanced covariates, two showed correlation $>0.2$ in absolute value, with at least one covariate with a standardized difference $>5 \%$ : WOMAC score was correlated with SF-12 physical score $(r=-0.513)$ and PEL was correlated with sex $(r=0.289)$ and WOMAC score $(r=-0.245)$. Therefore, we removed only the global assessment of the disease status, the current use of SYSADOA and the use of walking sticks from the PS model. The estimated $c$-statistic for the PS model with 23 covariates remained 0.684 . However, the provided threshold value was 0.682 . Consequently, after a preselection of covariates, a baseline imbalance was detected. This example also showed that our selection method allow for retaining a large amount of covariates, keeping the advantage of a global method over univariate testing. In the original paper, authors used an Inverse Probability of Treatment Weighted (IPTW) estimator to correct for baseline imbalance.

\section{Discussion}

In this paper, we provide a new tool, based on the $c$-statistic of the PS model, to detect baseline imbalance in CRTs. This tool performed well for CRTs with a large sample size and a large number of covariates and allowed allowed us to capture global information, in contrast to univariate tests. In the first motivating example, our method revealed a predictor of intervention allocation that univariate methods ignored, and confirmed the presence of imbalance and the requirement of adjusted statistical methods when estimating the intervention effect. The efficiency of the proposed preselection strategy was shown in the second motivating example. Even if a subset of covariates was retained, the subset was still meaningful for a global approach because the pre-selection method aimed at retaining the correlation patterns between covariates.

In practice, this approach can be viewed as a kind of hypothesis testing because it relies on a "known" probability distribution and uses a threshold value defined according to a significance level (5\% in our study because we used the $95^{\text {th }}$ percentile of the $c$-statistic distribution). Of note, we used the $95^{\text {th }}$ percentile of the $c$-statistic distribution under the hypothesis of no systematic imbalance to allow us to compare the results with classical univariate tests; however, to detect smaller baseline imbalances, smaller percentiles could be adopted, especially in CRTs with a large sample size with less chance variation in baseline covariates expected. Indeed, adjustment on balanced covariates does not have a negative impact such as omitting an unbalanced risk factor would, and thus this method will be less restrictive with a smaller percentile. Moreover, as for classical tests for which a $p$-value close to $5 \%$ has to be interpreted carefully, estimated c-statistics close to their threshold values do not necessarily mean that there is no confounding bias (if $c<$ threshold) or a systematic bias (if $c>$ threshold). In these situations, a risk of bias can be suspected and further considerations about the link between covariates and outcome are needed to assess the risk of bias. But again, unnecessary adjustment would have a smaller impact on the analysis that 
the omission of a confounder in the analysis. Statistical testing is not recommended in individually randomized trials because they are not theoretically prone to confounding bias [11]. However, as previously explained, this assumption does not hold in CRTs that randomize clusters before selecting participants. Therefore, the quantitative approach proposed in this paper could be useful to improve both the reporting of baseline characteristics and the subsequent statistical analysis.

The performance of our method was high for $n=$ 500 , a sample size close to that observed in practice (the interquartile range of sample size per arm being [143-866]) in a recent systematic review [42]. For $n=$ 100 , i.e. a value below the first quartile of the observed sample size per arm, the performance was low or moderate. In these situations that are highly prone to chance imbalance, covariate adjustment may be useful even if our method does not lead to the conclusion of a baseline imbalance. Our method must be viewed first as a tool to assess the risk of confounding bias and then to help identify CRTs in which an adjustment is needed, but for small sample sizes, covariate adjustment should be systematic, considering the high risk of sample fluctuations.

A limitation of this tool is the focus on 'overt bias' only, i.e. it can only assess the imbalance on observed characteristics as defined by Rosenbaum [49]. However, most trials collect information on a large number of baseline covariates, and given the fact that there are likely to be associations between different covariates, it is unlikely for the observed baseline covariates to be balanced between treatment arms, but for the unobserved covariates to be imbalanced. This would only happen if the observed and unobserved covariates were independent from each other and the association of these variables with the outcome variable is weak. Moreover, this tool can only help in assessing confounding bias, but not selection bias (i.e. difference in characteristics between recruited and not recruited patients). In order to detect selection bias, baseline characteristics of patients not recruited would be necessary, such as screen log data, but these data are often not available.

Further research is needed to assess the performance of the proposed method in a wider variety of situations. This study focused mainly on individual baseline characteristics: because clusters are the randomization unit, systematic imbalance on cluster-level covariates should not occur, provided the randomization method has been implemented correctly with appropriate allocation concealment, but chance imbalance on these covariates may occur. In particular, chance imbalance is likely to occur with only few randomized clusters, which is frequent; a systematic review showed that the median number of randomized clusters is 34 [9].

\section{Conclusion}

To avoid a risk of confounding bias, CRTs should, if possible, be designed to respect the usual chronology of randomized trials (recruitment and then randomization of clusters). However it is not always feasible in practice, for example when participants are incident cases. When clusters are randomized prior to participants being recruited, the proposed method is a helpful qualitative tool to assess the risk of bias in CRTs and to provide guidance for covariate adjustment.

\section{Additional file}

Additional file 1: Appendix. Simulation plan and distribution of the c-statistic without baseline imbalance. The R code to compute threshold values is available on request to the corresponding author. (PDF $279 \mathrm{~Kb}$ )

\section{Competing interests}

The authors declare that they have no competing interests.

\section{Authors' contributions}

$C L, A C$ and $B G$ conceived the study. $C L$ performed the simulation study and $C L, A C, Y F$ and $B G$ interpreted the results. $C L, A C, Y F$ and $B G$ drafted the manuscript. All authors read and approved the final manuscript.

\section{Acknowledgements}

The authors are grateful to Jerry Cottrell, Professor Olivier Chosidow and Professor Philippe Ravaud for granting permission to use their data.

\section{Author details}

${ }^{1}$ INSERM U1153, Paris, France. ${ }^{2}$ INSERM CIC 1415, Tours, France. ${ }^{3}$ CHRU de Tours, Tours, France. ${ }^{4}$ Department of Medical Statistics, London School of Hygiene and Tropical Medicine, London, United Kingdom. ${ }^{5}$ Université François-Rabelais, PRES Centre-Val de Loire Université, Tours, France. ${ }^{6}$ SPHERE (EA 4275): Biostatistics, Clinical Research and Subjective Measures in Health Sciences, Université de Nantes, Nantes, France.

Received: 2 June 2015 Accepted: 8 December 2015

Published online: 22 January 2016

\section{References}

1. Donner A, Klar N. Design and Analysis of Cluster Randomization Trials in Health Research. London: Wiley; 2000.

2. Torgerson DJ, Torgerson C. Designing Randomised Trials in Health, Education and the Social Sciences: an Introduction. Basingstoke: Palgrave Macmillan; 2008.

3. Carter B. Cluster size variability and imbalance in cluster randomized controlled trials. Stat Med. 2010;29(29):2984-93.

4. Hahn S, Puffer S, Torgerson DJ, Watson J. Methodological bias in cluster randomised trials. BMC Med Res Method. 2005;5(1):10.

5. Eldridge S, Ashby D, Bennett C, Wakelin M, Feder G. Internal and external validity of cluster randomised trials:s systematic review of recent trials. BMJ. 2008;336(7649):876-80.

6. Puffer S, Torgerson D, Watson J. Evidence for risk of bias in cluster randomised trials: review of recent trials published in three general medical journals. BMJ. 2003;327(7418):785-9.

7. Brierley G, Brabyn S, Torgerson D, Watson J. Bias in recruitment to cluster randomized trials: a review of recent publications. J Eval Clin Pract. 2012;18(4):878-86.

8. de Hoop E, Teerenstra S, van Gaal BG, Moerbeek M, Borm GF. The "best balance" allocation led to optimal balance in cluster-controlled trials. J Clin Epidemiol. 2012;65(2):132-7.

9. Eldridge SM, Ashby D, Feder GS, Rudnicka AR, Ukoumunne OC. Lessons for cluster randomized trials in the twenty-first century: as systematic review of trials in primary care. Clinical Trials (London, England). 2004;1(1): 80-90. 
10. Ivers NM, Halperin IJ, Barnsley J, Grimshaw JM, Shah BR, Tu K, et al. Allocation techniques for balance at baseline in cluster randomized trials: a methodological review. Trials. 2012;13(1):120.

11. Stang A, Poole C, Kuss O. The ongoing tyranny of statistical significance testing in biomedical research. Eur J Epidemiol. 2010;25(4):225-30.

12. Senn S. Seven myths of randomisation in clinical trials. Stat Med. 2013;32(9):1439-50.

13. Moher D, Schulz KF, Altman D. The CONSORT statement: Revised recommendations for improving the quality of reports of parallel-group randomized trials 2001. EXPLORE: J Sci Healing. 2005;1(1):40-5.

14. Campbell MK, Elbourne DR, Altman DG. CONSORT statement: extension to cluster randomised trials. BMJ. 2004;328(7441):702-8.

15. Fayers PM, King M. A highly significant difference in baseline characteristics: the play of chance or evidence of a more selective game? Qual Life Res. 2008;17(9):1121-1123.

16. Wright N, Ivers N, Eldridge S, Taljaard M, Bremner S. A review of the use of covariates in cluster randomized trials uncovers marked discrepancies between guidance and practice. 2014. doi:10.1016/j.jclinepi.2014.12.006.

17. Giraudeau B, Ravaud P. Preventing bias in cluster randomised trials. PLoS Med. 2009;6(5):1000065

18. Franklin JM, Rassen JA, Ackermann D, Bartels DB, Schneeweiss S. Metrics for covariate balance in cohort studies of causal effects. Stat Med. 2013. doi:10.1002/sim.6058.

19. Rosenbaum PR, Rubin DB. The bias due to incomplete matching. Biom;41(1):103-16.

20. Smirnov N. Table for estimating the goodness of fit of empirical distributions. Ann Math Stat. 1948;19(2):279-81.

21. Thompson JW. A note on the lévy distance. J Appl Prob. 1975;12(2):412-4.

22. Belitser SV, Martens EP, Pestman WR, Groenwold RHH, de Boer A, Klungel $\mathrm{OH}$. Measuring balance and model selection in propensity score methods. Pharmacoepidemiol Drug Saf. 2011;20(11):1115-1129.

23. Ali MS, Groenwold RHH, Pestman WR, Belitser SV, Roes KCB, Hoes AW, et al. Propensity score balance measures in pharmacoepidemiology: a simulation study. Pharmacoepidemiol Drug Saf. 2014. doi:10.1002/pds.3574.

24. Mahalanobis P. On the generalised distance in statistics. In: Proceedings National Institute of Science, India, Vol. 2, No. 1; (16 April 1936). p. 49-55.

25. lacus SM, King G, Porro G. Multivariate matching methods that are monotonic imbalance bounding. J Am Stat Assoc. 2011;106(493): 345-61.

26. Weitzen S, Lapane KL, Toledano AY, Hume AL, Mor V. Weaknesses of goodness-of-fit tests for evaluating propensity score models: the case of the omitted confounder. Pharmacoepidemiol Drug Saf. 2005;14(4): 227-38.

27. Leyrat C, Caille A, Donner A, Giraudeau B. Propensity scores used for analysis of cluster randomized trials with selection bias: a simulation study. Stat Med. 2013;32(19):3357-372.

28. Leyrat C, Caille A, Donner A, Giraudeau B. Propensity score methods for estimating relative risks in cluster randomized trials with low-incidence binary outcomes and selection bias. Stat med. 2014. doi:10.1002/sim.6185. PMID: 24771662.

29. Ravaud P, Giraudeau B, Logeart I, Larguier JS, Rolland D, Treves R, et al. Management of osteoarthritis (OA) with an unsupervised home based exercise programme and/or patient administered assessment tools. a cluster randomised controlled trial with a $2 \times 2$ factorial design. Ann Rheum Dis. 2004;63(6):703-8.

30. Ravaud P, Flipo RM, Boutron I, Roy C, Mahmoudi A, Giraudeau B, et al. ARTIST (osteoarthritis intervention standardized) study of standardised consultation versus usual care for patients with osteoarthritis of the knee in primary care in france: pragmatic randomised controlled trial. BMJ. 2009;338:b421.

31. Rosenbaum PR, Rubin DB. The central role of the propensity score in observational studies for causal effects. Biometrika. 1983;70(1):41-55.

32. $\mathrm{Xu} \mathrm{Z}$, Kalbfleisch JD. Propensity score matching in randomized clinical trials. Biometrics. 2010;66(3):813-23.

33. Harrell F. Regression Modeling Strategies : with Applications to Linear Models, Logistic Regression, and Survival Analysis. New York: Springer; 2001.

34. Altman D. Practical Statistics for Medical Research, 1st edn. London: New York: Chapman and Hall; 1991.
35. Westreich D, Cole SR, Funk MJ, Brookhart MA, Stürmer T. The role of the c-statistic in variable selection for propensity score models. Pharmacoepidemiol Drug Saf. 2011;20(3):317-20.

36. Turner RM, White IR, Croudace T. Analysis of cluster randomized cross-over trial data: a comparison of methods. Stat Med. 2007;26(2): 274-89.

37. Kul S, Vanhaecht K, Panella M. Intraclass correlation coefficients for cluster randomized trials in care pathways and usual care: hospital treatment for heart failure. BMC health Serv Res. 2014;14(1):84.

38. Kimko H, Duffull SB. Simulation for Designing Clinical Trials: A Pharmacokinetic-Pharmacodynamic Modeling Perspective. New York: CRC Press; 2002.

39. Perkins SM, Tu W, Underhill MG, Zhou XH, Murray MD. The use of propensity scores in pharmacoepidemiologic research. Pharmacoepidemiol Drug Saf. 2000;9(2):93-101.

40. Brookhart MA, Schneeweiss S, Rothman KJ, Glynn RJ, Avorn J, Stürmer T. Variable selection for propensity score models. Am J Epidemiol. 2006;163(12):1149-1156

41. Kuss $O$. The $z$-difference can be used to measure covariate balance in matched propensity score analyses. J Clin Epidemiol. 2013;66(11): 1302-1307.

42. Ivers NM, Taljaard M, Dixon S, Bennett C, McRae A, Taleban J, et al. Impact of CONSORT extension for cluster randomised trials on quality of reporting and study methodology: review of random sample of 300 trials, 2000-8. BMJ. 2011;343:5886-886.

43. Greenland S, Robins JM, Pearl J. Confounding and collapsibility in causal inference. Stat Sci. 1999;14(1):29-46.

44. Murray DM, Blitstein JL. Methods to reduce the impact of intraclass correlation in group-randomized trials. Eval Rev. 2003;27(1):79-103.

45. Gomes M, Grieve R, Nixon R, Ng ES-W, Carpenter J, Thompson SG. Methods for covariate adjustment in cost-effectiveness analysis that use cluster randomised trials. Health Econ. 2012;21(9):1101-1118.

46. van Marwijk HW, Ader H, de Haan M, Beekman A. Primary care management of major depression in patients aged 55 years:. Br J Gen Prac. 2008;58:680-7.

47. Taft AJ, Small R, Hegarty KL, Watson LF, Gold L, Lumley JA. Mothers' AdvocateS in the community (MOSAIC)-non-professional mentor support to reduce intimate partner violence and depression in mothers: a cluster randomised trial in primary care. BMC public health. 2011;11:178.

48. Patrick AR, Schneeweiss S, Brookhart MA, Glynn RJ, Rothman KJ, Avorn J, et al. The implications of propensity score variable selection strategies in pharmacoepidemiology: an empirical illustration. Pharmacoepidemiol Drug Saf. 2011. doi:10.1002/pds.2098.

49. Rosenbaum PR. Discussing Hidden Bias in Observational Studies. Ann Intern Med. 1991;115(11):901-5.

50. Austin PC. Balance diagnostics for comparing the distribution of baseline covariates between treatment groups in propensity-score matched samples. Stat Med. 2009;28(25):3083-107.

51. Austin PC. Using the standardized difference to compare the prevalence of a binary variable between two groups in observational research. Commun Stat Simul Comput. 2009;38(6):1228-1234.

\section{Submit your next manuscript to BioMed Central and we will help you at every step:}

- We accept pre-submission inquiries

- Our selector tool helps you to find the most relevant journal

- We provide round the clock customer support

- Convenient online submission

- Thorough peer review

- Inclusion in PubMed and all major indexing services

- Maximum visibility for your research

Submit your manuscript at www.biomedcentral.com/submit 